\title{
An Energy Based Analysis of the Quark/Hadron Impact on Cosmic Decoupling
}

\author{
Thomas A. Kriz \\ Advanced Studies, Alpha Omega Research Foundation, Cedar Park, USA
}

\section{Email address: \\ t_kriz@yahoo.com}

\section{To cite this article:}

Thomas A. Kriz. An Energy Based Analysis of the Quark/Hadron Impact on Cosmic Decoupling. American Journal of Modern Physics. Vol. 6, No. 6, 2017, pp. 148-152. doi: 10.11648/j.ajmp.20170606.16

Received: June 4, 2017; Accepted: June 19, 2017; Published: November 10, 2017

\begin{abstract}
The initial state of the cosmos is analytically modeled as a radiation filled spherical cavity that expands from a singularity to later act as a clock and energy source in support of a 3-stage, radiation to a quark-hadron based, decoupling process. The model thereby avoids a need for Inflation and the presence of matter at start-up and during the radiation dominated phase, but nevertheless remains strongly consistent with attributes of the Guth Inflationary model. At decoupling, only quark family \#1 with up-down attributes has adequate energy to successfully complete decoupling. Earlier in a 3 -stage process, attempts at hadronization by quark families \#2 and \#3 fail due to large quark size and binding energy requirements that exceed the available radiation energy supply. These attempts decay rapidly to take a quark family \#1 form. Decoupling is further modeled as half-spin based radiation resonance forms that are linked, via particle time dilation, to matter based micro-black-holes.
\end{abstract}

Keywords: Cosmic Decoupling, Expanding Radiation Filled Spherical Cavity, Quark Families, Hadron Decay

\section{Introduction}

A recently published paper on quark family attributes allows one to see more clearly their impact on cosmic evolution and decoupling.

[1] The following chart (Table 1) where $\mathrm{J}$ is total angular momentum, B is baryon number, $\mathrm{Q}$ is electric charge, and $\mathrm{I}_{3}$ is Isospin summarizes a list of relevant observed quark attributes from that paper which will be used here to analyze the role of quarks at various stages of cosmic development. Since quarks are always confined within hadrons, the chart data is indirectly derived from observations on particle based hadron collider experiments. Note, also, that current astronomy based surveys on the spectral attributes of matter elsewhere in the cosmos, so far, also confirms that no stable hadron types exist other than those from family \#1. Recall then that a hadron particle such as a neutron can be viewed, from a quark viewpoint, as composed of two Downs and one Up, and a proton, as one Down and two Ups. Note also that an isolated neutron particle set only has a life span of 889 seconds [2] due to Beta decay. But, by comparison, both the Charm-Strange family and especially the Top-Bottom family have significantly shorter life spans $[3,4,5$, 6] and can be viewed as failed attempts to successfully hadronize during a 3 -stage cosmic decoupling process.
Table 1. Quark Family Attributes.

\begin{tabular}{lllllll}
\hline Fam & Name & Mass $\left(\mathbf{M e v} / \mathbf{c}^{2}\right)$ & $\mathbf{J}$ & $\mathbf{B}$ & $\mathbf{Q}$ & $\mathbf{I}_{\mathbf{3}}$ \\
\hline \multirow{2}{*}{ st } & Up & $3 \pm 0.7 \pm 0.5$ & $1 / 2$ & $+1 / 3$ & $+2 / 3$ & $+1 / 2$ \\
& Down & $4.8 \pm 0.5 \pm 0.3$ & $1 / 2$ & $+1 / 3$ & $-1 / 3$ & $-1 / 2$ \\
\multirow{2}{*}{ nd } & Charm & $1275 \pm 25$ & $1 / 2$ & $+1 / 3$ & $+2 / 3$ & 0 \\
& Strange & $95 \pm 5$ & $1 / 2$ & $+1 / 3$ & $-1 / 3$ & 0 \\
3 3rd & Top & $173210 \pm 1220$ & $1 / 2$ & $+1 / 3$ & $2 / 3$ & 0 \\
& Bottom & $4180 \pm 30$ & $1 / 2$ & $+1 / 3$ & $-1 / 3$ & 0 \\
\hline
\end{tabular}

In this regard, see also the lepton life spans listed below in a second associated chart (Table 2) that summarizes the spin-1/2 lepton family particle attributes that are part of the atomic particle set that includes the above defined quark families. [7] The lepton life span data listed here gives a close approximation of the Charm-Strange and Top-Bottom quark family group attributes during hadronization attempts.

Table 2. Lepton Family Attributes.

\begin{tabular}{llll}
\hline Family & Name & Mass $\left(\right.$ Mev/c $\left.\mathbf{c}^{\mathbf{2}}\right)$ & Mean Life \\
\hline 1st & Electron & 0.511 & stable \\
2nd & Muon & 106 & $2.2 \times 10^{-6} \mathrm{~s}$ \\
3rd & Tau & 1784 & $3.4 \times 10^{-23} \mathrm{~s}$ \\
\hline
\end{tabular}




\section{Energy Based Analysis}

The cosmic matter formation scenario in use here assumes that the neutron particles develop first, and then, via Beta decay, turn into proton/electron sets that further leads to formation of Hydrogen particles. In order to see the need for a 3 -stage decoupling process in this scenario, it is first necessary to examine the energy requirements of the quark family attributes listed above for neutron style particle generation. Assuming a first letter symbol for each quark type, the respective quark constituents for each family during the neutron-style hadronization attempts, are udd, css, and tbb. The following chart (Table 3 ), in reverse cosmic time order, then gives a list of the approximate neutron hadronization attempt attributes during decoupling. This chart also assumes that radiation level at the start of decoupling has an equivalent mass property defined by [8]

$$
\mathrm{m}=\omega \mathrm{h} / \mathrm{c}^{2}=2 \pi \mathrm{fh} / \mathrm{c}^{2}
$$

where $\omega$ is angular frequency, $f$ is frequency defined in Hertz units, h is Planck's constant, and c is the speed of light. Note also that the chart does not include binding energy for a neutron particle. If the 1-Up/2-Down quark set in quark family \#1 for a neutron included the binding energy, the total energy requirement [9] would be $939.57 \mathrm{Mev} / \mathrm{c}^{2}$ at an equivalent cosmic radiation frequency of $2.27 \times 10^{23} \mathrm{~Hz}$. It can be seen then that the neutron binding energy requirement is equivalent to $927.67 \mathrm{Mev} / \mathrm{c}^{2}$, or approximately an amount equal to three orders of magnitude larger than the $11.9 \mathrm{Mev} / \mathrm{c}^{2}$ value for neutron particle mass listed above.

Table 3. Staged Hadronization Activity.

\begin{tabular}{llll}
\hline Family & Order & Mass $\left(\right.$ Mev/c $\left.{ }^{2}\right)$ & Mean Life \\
\hline 1st & 3 & 11.9 & $2.66 \times 10^{20}$ \\
2nd & 2 & 465 & $3.54 \times 10^{22}$ \\
3rd & 1 & 181570 & $4.38 \times 10^{24}$ \\
\hline
\end{tabular}

Note also there is the same harmonic-like increase of 123 (or slightly more than two orders of magnitude) both in quark mass as well as radiation frequency and therefore also radiation energy supply between families. This shows that the radiation energy supply during families \#2 an \#3 hadronization attempts is only marginally sufficient to satisfy the energy requirements of the hadron particle itself, but is inadequate for binding energy requirements. As stated above, however, the total binding energy requirement for a neutron in the \#1 quark family is an additional $927.67 \mathrm{Mev} / \mathrm{c}^{2}$, about three orders of magnitude greater than that of the mass 11.9 $\mathrm{Mev} / \mathrm{c}^{2}$ of the neutron particle, and must also be supplied by radiation. It is worth noting then that this binding energy value is almost as large as that typically quoted for heavy atomic nuclei. For example, the nucleus of a gold $\mathrm{au}_{200}$ atom with 200 nucleons only requires a binding energy of [10]

$$
7.8(200 \text { nucleons })=1560 \mathrm{Mev}
$$

because of the large population of neutrons distributed in its nucleus significantly reduces the need for a large binding energy, as in the case of the quark set cited above. Thus, the very low mean life-span associated with failed neutron-style hadronization attempts found in the $2^{\text {nd }}$ and especially the $3^{\text {rd }}$ quark family is due to an inability to satisfy the total energy requirement that increases (by about five orders of magnitude) at each of those two stages. Note also, there currently exists no collider based experimental evidence that quark or hadron matter exists at energy levels beyond that of the $3^{\text {rd }}$ quark family, nor theoretically should there be any due to the high energy requirements. In fact, even though the existence of the Bottom quark was verified in 1977, [3] the existence of the Top quark, though very large but with a very short life-span, was not verified until 1995. [4, 5] This therefore implies that, prior to decoupling, the cosmic radiation dominated phase should not contain any quark or hadron matter, as have been suggested in several published references. [11, 12] The data given above also indicates that the 3 -stage decoupling process is not complete until the total accumulated neutron particle energy during decoupling has reached a $939.57 \mathrm{Mev} / \mathrm{c}^{2}$ level. This only occurs at the completion of the 3 -stage quark based decoupling process described above.

\section{Attributes of a Radiation Filled Expanding Spherical Resonator}

The previous section then requires that, before cosmic decoupling, the radiation dominated phase be represented by an expanding radiation filled spherical resonator with no matter. For boundary conditions, it must be center fed, open-circuited, start with an initial radiation wavelength that is the shortest possible increment of length, and expand at the speed of light. The next challenge then is to determine operating mode of such a resonator.

A suitable model for an expanding radiation dominated cosmos can be found in Ishii's text on Microwave Engineering. [13] As shown in that text, such a model is an open circuited, center-fed, loss-less radiation filled spherical cavity, with series style resonance with $\mathrm{n}=0$. The model is also expanding at a light speed rate, with radial wavelength set to Planck Length initially, and follows a simple form of Maxwell's Equations defined by the equation pair

$$
\begin{aligned}
& \nabla^{2} \mathrm{E}+\omega^{2} \epsilon_{0} \mu_{0} \mathrm{E}=0 \\
& \boldsymbol{\nabla}^{2} \mathrm{H}+\omega^{2} \epsilon_{0} \mu_{0} \mathrm{H}=0
\end{aligned}
$$

where electric field intensity $\mathrm{E}=\mathrm{E}_{0} \mathrm{e}^{\mathrm{i} \omega \mathrm{t}}$ and magnetic field intensity $\mathrm{H}=\mathrm{H}_{0} \mathrm{e}^{\mathrm{i} \omega t}, \mathrm{i}=(-1)^{1 / 2}$, $\omega$ is angular frequency, $\epsilon_{0}$ is the dielectric constant of free space, $\mu_{0}$ is the permeability of free space. The operating mode of the sphere can then be set to expand with a wavelength property as

$$
\lambda=c^{\prime} \operatorname{tr} / 4=c^{\prime} t L_{P} / 4
$$

where $c^{\prime}$ is a speed of light expansion rate, $t$ is time in seconds, $\mathrm{r}$ is radius of the sphere and $\lambda$ is radiation wavelength, initially set to Planck Length $\mathrm{L}_{\mathrm{P}}=1.616 \times 10^{-35} \mathrm{~m}$, Then, assuming that cosmic decoupling occurs at approximately [14] 


$$
\mathrm{t}=200,000 \mathrm{yrs}=6.31 \times 10^{12} \mathrm{~s},
$$

the wavelength of the radiation in the expanding sphere at this time is

$$
\lambda=\mathrm{c}^{\prime} \mathrm{tL} / 4=\left(3.00 \times 10^{8} / \mathrm{s}\right)\left(6.31 \times 10^{12} \mathrm{~s}\right)\left(1.616 \times 10^{-35} \mathrm{~m}\right) / 4=1.22 \times 10^{-15} \mathrm{~m} .(7)
$$

Thereby, it can be seen then that the wavelength associated with $\lambda$, at the decoupling stage of expansion, is approximately the size of a neutron particle, and the cosmic radiation frequency is approximately

$$
\left.\mathrm{f}=\mathrm{c}^{\prime} \lambda^{-1}=6.00 \times 10^{8} \mathrm{~m} / \mathrm{s}\right)\left(1.22 \times 10^{-15} \mathrm{~m}\right)^{-1}=2.46 \times 10^{23} \mathrm{~Hz},
$$

which is close to the above stated cosmic radiation frequency value for the total energy of a neutron.

The harmonic-like 123 quark mass growth factor cited above, between the adjacent quark families, shows how quark mass is somehow coupled to cosmic radiation frequency. Such an attribute can be treated mathematically as a radiation based modulation-like boundary condition.

\section{Particles Modeled as Radiation Resonance Based Micro-Black-Holes}

The previous section describes the initial stage of cosmic evolution in terms of a cavity like resonator model for the radiation-dominated phase. Matter formation can only occur after a 3-stage quark decoupling process that acts as a trigger for the start of a rapidly formed half-spin matter dominated phase. A remaining unanswered question here, however, concerns the process by which radiation is actually converted to matter form by the quark based decoupling trigger. A proposed conversion process, summarized here, can be found in a previously published paper by the Alpha Omega group. [15]

In that paper, it is shown that the energy conversion process in a cosmic expansion scenario, initially in a radiation dominated phase that transits to a half spin particle matter dominated phase, can be theoretically represented as a half-spin radiation resonance process defined by

$$
\beta=1 / 2 \text {, a spin particle with } \mathrm{v}=\mathrm{v}_{\mathrm{g}}=\mathrm{c} / 2, \mathrm{v}_{\mathrm{p}}=\mathrm{c}^{2} / \mathrm{v}_{\mathrm{g}}=2 \mathrm{c}, \xi^{2}=-3 / 4
$$

where $\beta$ is a spin attribute, $v_{p}$ and $v_{g}$ are respectively phase wave and group wave velocity of a wave packet focused set of electromagnetic harmonic components, $\mathrm{c}$ is the velocity of light, and defines the relativistic relationship between $\xi$ and $\beta$. The spin categories defined by $\beta=1,1 / 2,0$ assume torquing velocity components $\mathrm{v}_{\mathrm{g}}$ have a form which supports spin.

$$
\xi^{2}=\beta^{2}\left[1-(\mathrm{v} / \mathrm{c})^{2}\right]=\beta^{2}-1
$$

The altered light speed attributes of the resonance set can then be linked to a particle based gravitational time dilation attribute defined by [16]

$$
\Delta \mathrm{v} / \mathrm{v}=\Delta \tau / \tau=\mathrm{GM}\left[1 / \mathrm{r}_{1}-1 / \mathrm{r}_{2}\right]=\mathrm{M} /\left(\mathrm{c}^{2} \mathrm{r}\right)=1 / 2
$$

where $\Delta \mathrm{v}$ is the downward shift in clock or EM frequency,
$\Delta \tau / \tau$ is the dilated time, $\mathrm{G}$ is the gravitational constant, $\mathrm{M}$ is the mass of a spherical particle, and $r$ is the radial position of the wave boundary relative to its origin with $r_{2}>r_{1}$. Such a matter formation process is also shown to have space-time signature in cgi units that is consistent with a General Relativity Schwarzschield micro-black-hole solution [17]

$$
\mathrm{r}=2 \mathrm{GM} / \mathrm{c}^{2} \text {. }
$$

When matter is modeled as a set of micro-black-holes, one can then also obtain the same physically relevant gravitational solutions as those from General Relativity. [18] In addition to Schwarzschield solution, these include gravitational time dilation, gravitational deflection of light, time retardation of light, perihelion attributes of an orbiting planet, and a Kerr style field solution for a rotating sphere with mass $M$ and angular momentum $\mathrm{J}$ where radial distance $\mathrm{r}>\mathrm{J} / \mathrm{M}$.

As mentioned, within this context, it is proposed that a likely radiation to primitive matter conversion scenario is one where neutrons form first, followed by protons and electrons that form as a result of Beta decay in an isolated neutron population, that then provides a basis for star formation via a fusion based Hydrogen to Helium process. More complex atomic structures then form later as a function of the size attributes in aging stellar bodies. [19] In this regard, note that our sun in old age will become a "red giant" star that ultimately turns into iron. [20]

\section{Conclusions}

The foregoing presentation begins with an analysis of observed quark attributes to determine what impact they might have on cosmic evolution and decoupling. It is shown thereby that cosmic decoupling can only occur when cosmic radiation frequency corresponds to approximately $2.27 \times 10^{23} \mathrm{~Hz}$. Because of a quark mass growth factor equal to 123 between the three known families, and a falling radiation energy level due to cosmic expansion, however, analysis shows that it is impossible to have stable quark and hadron formation during the radiation dominated phase before the above defined cosmic 3-stage decoupling event. Other than during decoupling, cosmic radiation energy levels during the radiation dominated phase are inadequate to support the hadronization particle binding energy requirements. It is also shown by use of an analytic model that a cosmic decoupling event with radiation frequency $2.27 \times 10^{23} \mathrm{~Hz}$ is consistent with a cosmic age of 200,000 years. It should also be noted that there is no report of observed evidence, astronomically, or from collider experiments, to support stable hadron formation by any means other than that by the \#1 quark family. The quark family \#2 and \#3 failed attempts at hadronization, that decay to a normal family \# 1 hadron, appear to be due an increasingly inadequate radiation energy supply, as one goes backwards in cosmic time.

The decoupling process is further represented as one in which half-spin based radiation resonance forms are linked, via particle based time dilation, to a matter based micro-black-hole form. Such a micro-black-hole, though very 
small but finite, can be related to point mass concepts that are found in both classical physics and quantum theory. In quantum electrodynamics, however, when developing a perturbation expansion result, point size can lead to unwanted fictitious infinities in higher order terms because of an implied zero distance between the perturbation series terms. It also is important to note that micro-black-holes differ from stellar gravitational based black holes supported by a large mass collective. [21] Whereas stellar gravitational black holes have no observable structure, both cosmological and quantum theory give evidence that shows an internal structure in micro-black-holes is necessary to support wavelike and atomic properties of particle based matter. Therefore, although micro-black-holes can collectively coalesce as atomic nuclei, they do not otherwise exhibit the stellar gravitational black hole habit to swallow up other nearby matter. Isolated neutron collective sets are also known to be unstable.

\section{Addendum: A Comparison with the Guth Inflationary Model}

As mentioned the Abstract, it is not necessary to include cosmic Inflation in the analysis presented here. To see this, it is useful to make a comparison of the Guth [22] cosmic scenario (that includes Inflation) with the scenario presented here based on observed quark based collider data. A summary of the Guth Inflationary scenario can also be found in the Beiser text. [23] As a starting point, the reader should note that both scenarios begin with a reference to Planck Length. The Guth scenario assumes an initial cosmic size equal to Planck Length $\left(\mathrm{eg} ; \sim 10^{-45} \mathrm{~m}\right)$. The Guth scenario then proceeds to describe the evolution of cosmic radius as a function of time, that includes an Inflationary increase of about $10^{30}$ in size at time $10^{-30} \mathrm{~s}$, a radiation dominated phase that is laden with matter, and ends finally with the start of the atomic era (eg; decoupling) at about $10^{13} \mathrm{~s}$, the same end point as in the analysis given here. By comparison, the analysis given here describes the cosmic evolution in terms of the radiation energy level, beginning with a wavelength equal to Planck Length, that is modified by cosmic expansion up to the point of the decoupling event. Since expansion proceeds at the speed of light during the radiation dominated phase and ends with the same $\left(\sim 10^{13} \mathrm{~s}\right)$ decoupling time, one can see that its scenario is comparable to that rendered by the assumed initial size condition, Inflation impact, and (matter laced) radiation dominated phase found in the Guth model. Note that decoupling occurs at the same cosmic time $\left(\sim 10^{13} \mathrm{~s}\right)$ in both models. The analysis given here, however, is based on observed collider data, provides more process level detail, and avoids the assumptions of the Guth model regarding Inflation and assumed matter conditions at start-up and during the radiation dominated phase. Note that in the Guth model, the presence of matter in the radiation dominated phase tends to slow the cosmic expansion rate, but is balanced by an initial Inflationary expansion surge to yield an equivalent overall expansion rate equal to the speed of light. In summary then, both models: 1) Start with Planck Length, 2) End at the same decoupling time $\left(\sim 10^{13} \mathrm{~s}\right)$, and 3$)$ Feature the same overall cosmic expansion rate.

\section{Acknowledgements}

The author wishes to express gratitude for the encouragement and critical review received during the earlier stages of the work present here received from E. J. Bacinich, Director of the Alpha Omega Research Foundation, and several other members of our group, now deceased: Prof. Behram Kursunoglu and Dr. Walter Rosenthal. Prof. Kursunoglu was the Director of the Theoretical Institute at the the University of Miami (FL), and pursued graduate studies at Cambridge University under P. A. M. Dirac. Dr. Walter Rosenthal was one of the last students to pursue graduate studies in physics at the University of Berlin under Max Planck.

\section{References}

[1] K. A. Olive et al., China Physics, C, 38 (9) (2014).

[2] A. Beiser, Concepts of Modern Physics (Fifth Edition), (McGraw Hill, N. Y. 1995) p. 482.

[3] S. W. Herb et al., Phys. Rev. Letters, 39 (5) 252 (1977).

[4] S. Abachi et al., Phys. Rev. Letters, 74 (13) 2422 (1995).

[5] F. Abe et al., Phys. Rev. Letters, 74 (14) 2626 (1995).

[6] K. W. Staley. The Evidence for the Top Quark, (Cambridge University Press 2004) pp. 31-33.

[7] A. Beiser, Concepts of Modern Physics (Fifth Edition), (McGraw Hill, N. Y. 1995) p. 482.

[8] A. Beiser, Concepts of Modern Physics (Fifth Edition), (McGraw Hill, N. Y. 1995). p. 83.

[9] A. Beiser, Concepts of Modern Physics (Fifth Edition), (McGraw Hill, N. Y. 1995). Phys. Const. (inside front cover).

[10] A. Beiser, Concepts of Modern Physics (Fifth Edition), (McGraw Hill, New York 1995) p. 399.

[11] A. Beiser, Concepts of Modern Physics (Fifth Edition), (McGraw Hill, New York 1995) p. 600.

[12] H. C. Ohanian and R. Ruffini, Gravitation And Spacetime (Second Edition) (Norton \& Co., N. Y. 1994) p. 592.

[13] T. K. Ishii, Microwave Engineering (Second Edition) (Harcourt Brace Jovanovitch, New York 1989) p. 137.

[14] H. C. Ohanian and R. Ruffini, Gravitation And Spacetime (Second Edition) (Norton \& Co., N. Y. 1994) p. 616.

[15] T. A Kriz, Am. Journal of Modern Phys., 4 (1) p. 10 (2015).

[16] H. C. Ohanian and R. Ruffini, Gravitation And Spacetime (Second Edition) (Norton \& Co., N. Y. 1994) p. 167.

[17] T. A Kriz, Am. Journal of Modern Phys., 4 (1) p. 11 (2015).

[18] T. A. Kriz, Phys. Essays, 24, p. 124 (2011). 
[19] K. E. Sakmann, et al., Astrophysical Journal, 418 p. 457 (1993).

[20] www.space.com/22471-red-giant-stars.html

[21] T. A Kriz, Am. Journal of Modern Phys., 4 (1) p. 13 (2015).
[22] A. H. Guth, The Inflationary Universe, (Addison Wesley, New York 1997).

[23] A. Beiser, Concepts of Modern Physics (Fifth Edition), (McGraw Hill, New York 1995) pp. 504-505. 